\title{
Real-Time Digital Biometric Monitoring during Elite Athletic Competition: System Feasibility with a Wearable Medical-Grade Sensor
}

\author{
Mark A. Gorski ${ }^{\mathrm{a}}$ Stanley M. Mimoto ${ }^{\mathrm{a}}$ Vivek Khare $^{\mathrm{a}}$ Viprali Bhatkar $^{\mathrm{b}}$ \\ Arthur H. Combs ${ }^{c}$ \\ a Sports Data Labs Inc., Detroit, MI, USA; ${ }^{\mathrm{b}}$ Independent Consultant, Arlington, MA, USA; \\ 'FutureTech Strategies Inc., Natick, MA, USA
}

\section{Keywords}

Digital biomarkers - Real-time monitoring - Wearable

sensors · Athletic performance

\begin{abstract}
Introduction: Real-time digital heart rate (HR) monitoring in sports can provide unique physiological insights into athletic performance. However, most HR monitoring of elite athletes is limited to non-real-time, non-competition settings while utilizing sensors that are cumbersome. The present study was undertaken to test the feasibility of using small, wearable medical-grade sensors, paired with a novel technology system, to capture and process real-time HR data from elite athletes during professional competition. Methods: We examined the performance of the BioStamp $n$ Point ${ }^{\circledR}$ sensor compared to the Polar chest strap HR sensor in 15 Professional Squash Association (PSA) tournament matches in 2019-2020. Fourteen male professional squash players volunteered for the study (age $=23.8 \pm 4.9$ years; height $=177.9 \pm 7.1 \mathrm{~cm}$; weight $=71 \pm 7.0 \mathrm{~kg}$ ), which was approved by the PSA in accordance with their Code of General Conduct and Ethics. Algorithms developed by Sports
\end{abstract}

Data Labs (SDL; Detroit, MI, USA) used proprietary data collection, transmission, and signal processing protocols to produce $\mathrm{HR}$ values in real-time during matches. We calculated the mean and maximum HR from both sensors and used widely accepted measures of agreement to compare their performance. Results: The system captured $99.8 \%$ of HR data across all matches (range 98.3-100\%). The BioStamp's mean HR was $170.4 \pm 20.3 \mathrm{bpm}$, while the Polar's mean HR was $169.4 \pm 21.7 \mathrm{bpm}$. Maximum HR ranged from 182 to $202 \mathrm{bpm}$ (Polar) and 185 to $203 \mathrm{bpm}$ (BioStamp). Spearman's correlation coefficient $\left(r_{s}\right)$ was $0.986(p<0.001)$, indicating a strong correlation between the 2 devices. The mean difference $(d)$ in HR was $1.0 \mathrm{bpm}$, the mean absolute error was $2.2 \mathrm{bpm}$, and the percent difference was $0.72 \%$, demonstrating high agreement between device measurements. Conclusions: It is feasible to accurately measure and monitor real-time HR in elite athletes during competition using BioStamp's and SDL's proprietary system. This system facilitates development and understanding of physiological digital biomarkers of athletic performance and physical and psychosocial demands in elite athletic competition.

(c) 2021 The Author(s)

Published by S. Karger AG, Basel karger@karger.com www.karger.com/dib

Karger $\stackrel{\text { ' }}{5}$

GOPEN ACCESS
(C) 2021 The Author(s)

Published by S. Karger AG, Basel

This is an Open Access article licensed under the Creative Commons Attribution-NonCommercial-4.0 International License (CC BY-NC) (http://www.karger.com/Services/OpenAccessLicense), applicable to the online version of the article only. Usage and distribution for commercial purposes requires written permission.
Arthur H. Combs

FutureTech Strategies Inc. 8 Park Avenue

Natick, MA 01760 (USA) art.futuretech@gmail.com 


\section{Introduction}

On July 24, 1969, Neil Armstrong became the first person in history to land a spacecraft on the moon. Armstrong's average heart rate (HR) throughout the Apollo 11 mission was 71 beats/min (bpm). However, during the lunar landing itself Armstrong's HR reached 150; a rate similar to Indy car drivers' HRs during professional motor races $[1,2]$. While space flight and racing environments are unique, these examples illustrate that under extreme and complex conditions other stimuli beyond physical demand can have considerable impacts on $\mathrm{HR}$, including the response to psychological, social, and emotional stressors [3-6]. Similarly, sports such as professional squash require elite athletes to perform in unique environments and under extreme conditions that induce responses affecting the athlete's physiological state. These include extreme kinematic motion, physical exertion, and psychological stressors [7, 8]. Monitoring athletes' HR in such competitive settings may reveal important insights into athletes' health and performance not available from prior research $[9,10]$.

To date, most research on HR in elite athletes has been conducted in laboratory or non-competitive settings even though it is well known that HR response and maximum HR differ between laboratory and competitive settings. Data from professional competition has the potential to better represent the physiologic responses of professional athletes, as well as associated outcomes, even compared to laboratory studies $[2,4,10-12]$. Moreover, HR data from competitive settings have not been available in realtime, thus limiting the potential to understand the athlete's physiology during competition and preventing realtime, physiological data-based performance adjustments.

Most prior research has employed cumbersome HR sensor systems that can impede athletic performance. For example, the Polar chest strap has been used in several studies [13], but it is not ideal for elite athletic competition. The strap itself may not be comfortable, may restrict breathing, may become malpositioned during competition or may be a conscious distraction. Additionally, the system has low sampling rates, limiting future insights such as HR variability (HRV) that require more granular data.

In addition to the biosensor itself, there are significant technological and operational barriers to real-time data monitoring in professional athletic venues. While a few studies have examined in-game HR response during elite athletic competition, to our knowledge extremely few have been conducted among professionals during competition and none have gathered and processed HR in real-time.

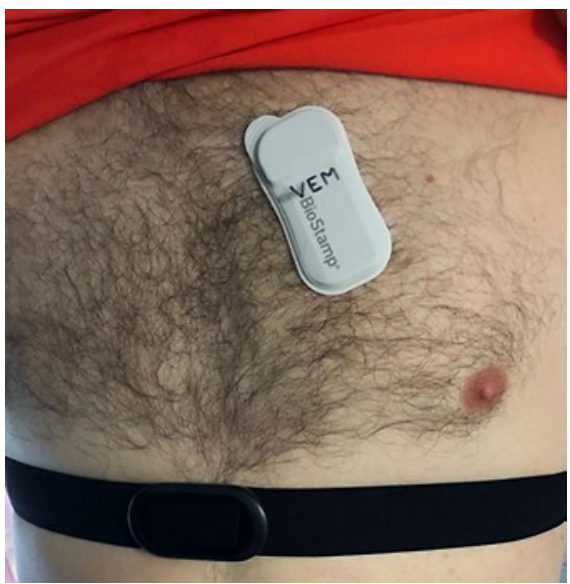

Fig. 1. BioStamp nPoint ${ }^{\circledR}$ and Polar chest strap positions on a study participant.

The present study was undertaken to test the feasibility of a novel data capture system designed to acquire and process real-time HR data from elite athletes during actual competition in professional venues. The system is comprised of a small wearable sensor (BioStamp ${ }^{\circledR}$ MC10 Inc., Lexington, MA, USA) and proprietary methods (Sports Data Labs [SDL] Inc., Detroit, MI, USA) for realtime communication with the wearable sensor, and for digital signal processing under conditions of extreme motion and physical exertion. This study was conducted to test data yield and quality, signal processing algorithm performance, and accuracy compared to a frequently used comparator device in a professional sports venue.

\section{Materials and Methods}

\section{Study Procedures}

Over the course of 3 Professional Squash Association (PSA) World Tour-sanctioned events at 3 different venues in 2019 and 2020, 14 male professional squash players volunteered to participate in the study, and data were collected during a single complete match in each of 13 players and 2 matches for 1 player $(n=15$ events). Study participants signed informed consent and waiver forms. The study was approved by the PSA in accordance with their Code of General Conduct and Ethics, and participants were paid USD 50 per match for their participation.

Prior to play, MC10 BioStamp $n$ Point ${ }^{\circledR}$ wearable sensors were applied by a research assistant in the ECG Lead II orientation on each participant's left chest with a BioStamp ${ }^{\circledR}$-specific adhesive (shown in Fig. 1). BioStamp nPoint ${ }^{\circledR}$ is an FDA $510(\mathrm{k})$ cleared, electrode-based, wearable, medical grade ECG sensor, with high sampling capabilities (configurable up to $1,000 \mathrm{~Hz}$ ). It was designed for use in clinical and home settings, where the activity of 
individuals is limited. It was selected for use in this study after discussions with professional sports athletes, governing organizations, and team owners because of its adhesive-based miniature form factor, comfort level, and minimal impact on athletic performance. The adhesive features an integrated hydrogel over the electrodes that enables consistent conductivity between skin and electrode. The adhesive was cleared by the US FDA during the $510(\mathrm{k})$ process having passed all required tests of biocompatibility and medical adhesive-induced skin injury. Furthermore, the adhesive was designed to wick sweat to avoid changes in signal quality. Participants were not required to shave their chests.

The research assistant also applied a Polar H10 chest strap device (Polar Electro Inc., Bethpage, NY, USA) around each participant's chest below the pectoral muscles. The Polar H10 is an ECGbased sensor, selected because it is the current accepted standard of commercially available HR monitors [13]. Both sensors were worn by the athletes continuously throughout match play.

Data Processing and Transmission

The BioStamp nPoint ${ }^{\circledR}$ generates raw analog front end sensor data from which a variety of real-time metrics can be derived, including HR, HRV, and surface electromyogram. For the purposes of this study, the analog data were processed to monitor HR throughout match play. Conversion of the raw analog data into a real-time HR was completed off-sensor via the SDL software system using SDL proprietary algorithms.

We integrated the BioStamp and Polar sensor with the SDL system to enable real-time, 2-way communication with, and data collection from, both sensors simultaneously. Data were streamed continuously throughout each match, and real-time HR for each study participant was monitored throughout match play. Both sensors transmitted data via Bluetooth Low Energy (BLE) communication. BLE was originally designed for short-distance communication ( $<100 \mathrm{~m}$ theoretical range). However, professional sports venues, including those used by the Professional Squash Association, often require sensors to communicate over longer distances, in hostile radio frequency environments, and the potential for significant motion interference. Such challenges can lead to signal degradation, preventing real-time data collection. Additionally, the competition venues can be populated by wireless communication-enabled mobile phones, wireless cameras and audio equipment, competing Wi-Fi equipment, and other networks and signals, creating additional sensor communication challenges. To address these issues, we created a proprietary transmission system including a high-gain antenna located near the field of play. Coupled with selected BLE transmission chipsets, we increased the communication distance, amplified the receiving connection, and minimized non-sensor signal interference.

\section{Data Alignment and Sampling}

Squash play consists of extreme motion, including significant lunging, bending, twisting, swinging, pivoting, and reaching that occur in very quick bursts. These types of movements, which result in increased pectoral muscle activity, increased movement of the skin during compressional and tensional stress, and increased movement of sensors, can create significant noise within the raw sensor data. To reduce data noise, SDL developed an algorithm based on the original Pan Tompkins method [14] with proprietary refinements and techniques, to convert the analog data into calculated $\mathrm{HR}$ values.

Athletic Competition Real-Time

Biometric Monitoring
Table 1. Athlete characteristics of $n=14$ male professional squash players

Participant characteristics

$\begin{array}{lc}\text { Mean age } \pm \text { SD, years } & 23.8 \pm 4.9 \\ \text { Hand of play } & \\ \quad \text { Right handed } & 13 \\ \text { Left handed } & 1 \\ \text { Mean height } \pm \text { SD, cm } & 177.9 \pm 7.1 \\ \text { Mean weight } \pm \text { SD, kg } & 71 \pm 7.0\end{array}$

In all testing cases, SDL received a processed HR in beats per minute (bpm) output from the Polar chest strap sensor at a rate of $1 \mathrm{~Hz}$ (once per second). The BioStamp ${ }^{\circledR}$ output was raw analog data that the system configured to send at a rate of $250 \mathrm{~Hz}$, subsequently producing an $\mathrm{HR}$ value once per second. Thus, each sensor was responsible for producing real-time $\mathrm{HR}$ values at $1 \mathrm{~Hz}$ (once per second) used for comparison and correlation analysis.

\section{Participants}

Fourteen male professional squash players volunteered to participate in the study (mean age $23.8 \pm 4.9$ years; mean height 177.9 $\pm 7.1 \mathrm{~cm}$; mean weight $71 \pm 7.0 \mathrm{~kg}$; Table 1 ). Informed consent was obtained prior to participation. Players were monitored from the start of play until the end of each match.

\section{Statistical Analysis}

Statistical analysis was performed using Stata software, version 15.0 (StataCorp, Houston, TX, USA). Minimum, mean, and maximum HR values (in bpm) were calculated continuously from both devices for each match, and overall. To examine the relationships between the measured HR from the Polar and BioStamp nPoint ${ }^{\circledR}$ sensors, widely used measures of agreement were calculated. Spearman's correlation $\left(r_{\mathrm{s}}\right)$ was performed to examine the correlation between the HR calculated by each device. The mean difference $(d)$, mean absolute error, percent difference, and Bland-Altman plots were performed to evaluate agreement between HR generated by each device. Errors-in-variables regression with bootstrapping and clustered robust standard errors were performed to estimate variance, generating the root mean square error (RMSE). This analytic approach was selected because both devices were subject to measurement error, and to account for measurement clustered within 15 matches. Statistical significance was set at $p<0.05$, and all tests were 2 -tailed.

\section{Results}

The purpose of the present study was to test the feasibility of a platform for measuring real-time biometrics in professional athletes during competition. The system consists of 3 components - the wearable sensor, the communications and data collection system, and the signal processing algorithms all operating in real-time. 


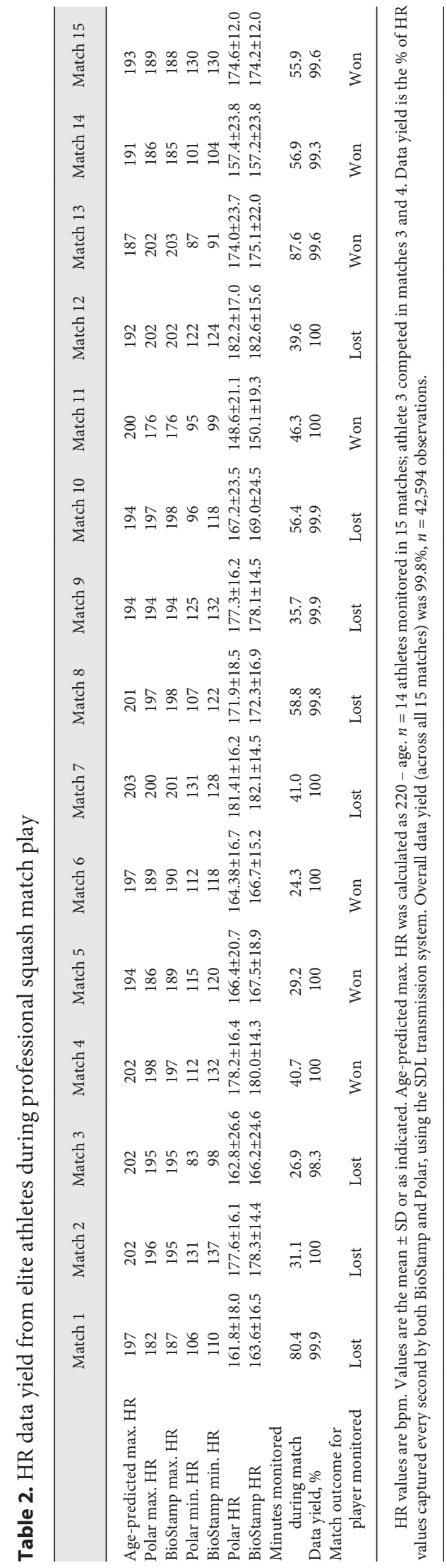

Table 2 summarizes the data yield of the entire system. Across the 15 matches HR data were available for 710.8 $\min$ or $42,648 \mathrm{~s}$. The total data yield (both sensors) was $99.8 \%$ and ranged from 98.3 to $100 \%$ (100\% was achieved in 7 of the 15 matches). Age-predicted estimated maximum HR was high (often exceeding $200 \mathrm{bpm}$ ) due to the young ages of the athletes, but interestingly was exceeded by the actual maximum HR in 3 athletes and identical in another.

Table 3 summarizes the comparison of the HR data acquired from the 2 sensors. The mean HR across all data was $170 \pm 20.3 \mathrm{bpm}$ for the BioStamp and $169.4 \pm 21.7$ bpm for Polar. The median HR was identical in both sensors at $176 \mathrm{bpm}$. Spearman's rho correlation coefficient $\left(r_{\mathrm{s}}\right)$ was 0.986 . The regression line shown in Figure 2 is close to the line of identity, with a mean absolute error of $2.2 \pm 2.5$, an RMSE of 1.946, and a percent error of $0.72 \pm$ $2.16 \%$. These data indicate a strong correlation and high agreement between HR measured by the BioStamp and Polar sensors and allow for the fact that either of the sensors could be responsible for any discordance.

A visual display of HR output from both sensors in one match is shown in Figure 3. The participant's HR varied widely, rapidly, and peaked intermittently throughout the match. Once play is initiated, HR varies from bursts of exertion to time between points, but remains predominantly above 140 during active match play, and averages $176 \mathrm{bpm}$.

\section{Discussion}

The primary purpose of the present study was to test the feasibility of a novel system for measuring real-time $\mathrm{HR}$ in professional athletes during competition. We compared the accuracy and data yield from the novel system to a simultaneous data stream from the frequently referenced Polar chest strap. In this feasibility study we demonstrated both excellent data yield and high correlation to an established HR monitor and the clear ability to capture real-time HR during elite athletic competition.

The results of this study have several implications. First, numerous studies have demonstrated that neither age-predicated maximum $\mathrm{HR}$ calculations nor maximum $\mathrm{HR}$ assessed by laboratory measurements of $\mathrm{VO}_{2}$ max accurately predicts maximum $\mathrm{HR}$ performance during competition [15-17]. Moreover, the exhaustive review by Robergs and Landwehr [18] documents the shortcomings of all published approaches to estimating maximum HR in the laboratory. 
Fig. 2. Regression analysis of HR values. Errors-in-variables regression with bootstrapping with clustered robust standard errors.

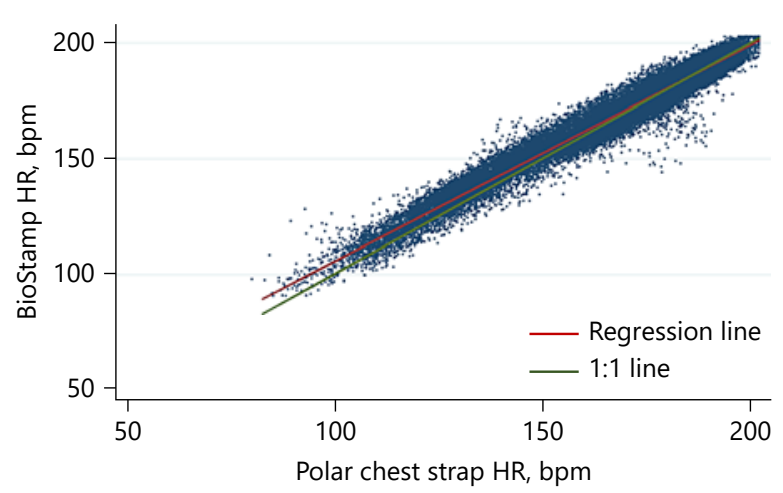

$N=15$ matches $n=42,594$ observations RMSE $=1.946$ Slope $=0.9367903$ Intercept $=11.71315$ $r^{2}=0.9908$
Table 3. Differences in measured HR using BioStamp and Polar sensors

\begin{tabular}{lc}
\hline Endpoint & HR, bpm \\
\hline Overall observed rate (min.-max.) & $82-203$ \\
BioStamp, HR & $170.4 \pm 20.3$ \\
Polar, HR & $169.4 \pm 21.7$ \\
BioStamp median HR & 176 \\
Polar median HR & 176 \\
Absolute error (BioStamp - Polar) & $2.2 \pm 2.5$ \\
Mean difference d (BioStamp - Polar) & $1.0 \pm 3.2$ \\
Mean difference, \% & $0.72 \pm 2.16$ \\
Spearman's rho correlation coefficient & 0.986 \\
RMSE & 1.946 \\
\hline
\end{tabular}

Values are the mean \pm SD or as indicated. Data collected from $n=15$ PSA matches in 2019-2020. RMSE, root mean square error.

The data from the present study clearly reveal that during elite squash competition athletes' HRs average 176 for nearly $45 \mathrm{~min}$. Moreover, maximum HR can exceed $200 \mathrm{bpm}$ and often exceeds either age- or laboratory-predicted maximum HR. The current study presents a system that solves the problem of relying on putative predictions by actually observing human athletic performance.

A second implication is that the HR of a given athlete at a given time has both a physical demand component and a psychosocial emotional component. Semin et al. [11], studying NCAA distance runners, found that laboratory predictions of HRmax (ventilation threshold, $\mathrm{VO}_{2} \max$ ) failed to predict maximum HR during active training and competition. Sanders et al. [10] found that in NCAA women's basketball players maximum HR varied by game period with time spent at $>85 \%$ maximum

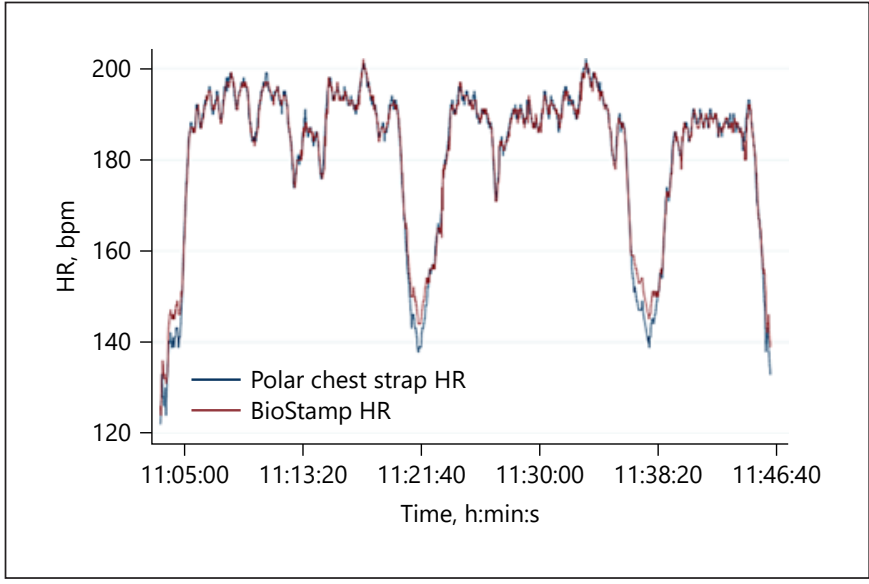

Fig. 3. BioStamp and Polar chest strap measurements in match 12.

HR being greatest in the fourth quarter when the game's outcome would be decided. Jacobs and Olvey [2], studying race car drivers, noted significantly different HR responses in drivers alone on a track, when other cars were present, and during active competition.

The current study demonstrates the precision of realtime HR observations, thus creating the opportunity to look more deeply into inter-beat intervals, HRV, indices of the stress response, and autonomic nervous system (ANS) activity during competition. Schmitt et al. [19] observed that the pattern of increased HR and decreased HRV during performance indicated fatigue, an observation that can be useful in athletic training. Buchheit [20] highlighted the importance of parsing the differences in HRV due to athletic activity and influence of the ANS. While gaining preliminary insights by measuring HRV before, during, and after competition, he reported that 
monitoring HR during competition was problematic precisely the problem the current study was designed to address.

A compelling study by Schwaberger [3] also examined 20 race car drivers during competition. He found that while race car driving has moderate physical demand, the principle influence on HR was psychological stress. The drivers' mean maximum HR was near $90 \%$ of that predicted by $\mathrm{VO}_{2}$ max despite only a moderate physical demand. Schwaberger [3] went on to measure the drivers' catecholamine excretion and found a $>2$-fold increase after a competitive race compared with baseline. Other stress-related hormonal (HGH, glucocorticoid) and biological indicators of metabolic stress (FFA, glucose levels) were also markedly elevated. The author concluded that the HR increase during race conditions was composed of 3:1 psychological to physical demand. One can infer that precise observations of real-time HR, HRV, and other digital biomarkers could help parse these influences on HR during athletic competition, giving the athlete the opportunity to mitigate influences like fatigue, stress, hydration, and game situation during a given match.

The limitations of the study include the small number of study subjects $(n=14)$, the lack of gender, age, or body habitus diversity, and the limitation to professional squash competition. While we look forward to the prospect of assessing the psychosocial influences on athletic performance, the present study was not designed to look at stress as an influence and athletes' insights in this regard were not solicited.

While the game of squash presents unique kinematic challenges, other sports may present others not tested here. Similarly, the ability to extend the system beyond a squash court to other sports venues like tennis courts, golf courses, stadiums, and arenas is unknown. The current results are nonetheless encouraging.

When Neil Armstrong's HR reached 150 as he landed the Lunar Module, the importance of real-time HR monitoring was reaffirmed. It also made it clear that HR and maximum $\mathrm{HR}$ are determined by many more factors than just physical demand. Psychological influences, the physiological stress response, fatigue, the state and influence of the ANS, situation specifics, and many other influences all determine real-time HR. There is an opportunity for future research with the current system to parse these influences, and to look at both sport-specific and athletespecific digital biomarkers that can help us better understand athletic training and performance, especially during real-time competition.

\section{Acknowledgements}

The authors wish to gratefully acknowledge Mary Findling $\mathrm{PhD}$ and Michael Findling for their invaluable contributions and Ellora Sen-Gupta for assistance with manuscript preparation. The authors also gratefully acknowledge the cooperation of the Professional Squash Association and the opportunity to conduct the study during their sanctioned professional events.

\section{Statement of Ethics}

Informed consent and the Code of General Conduct and Ethics of the Professional Squash Association (PSA) are referenced in the manuscript. Study participants signed informed consent and waiver forms. The study was approved by the PSA in accordance with their Code of General Conduct and Ethics.

\section{Conflict of Interest Statement}

M.A.G., S.M.M., and V.K. are co-founders of Sports Data Labs Inc., and have received equity compensation from Sports Data Labs Inc. Dr. Viprali Bhatkar is a paid algorithm consultant of Sports Data Labs Inc. Dr. Arthur H. Combs is a paid consultant of Sports Data Labs Inc.

\section{Funding Sources}

This study was funded by Sports Data Labs Inc.

\section{Author Contributions}

M.A.G. contributed to the in-venue research and writing of the manuscript. S.M.M. contributed to the in-venue research. V.K. contributed to algorithm development and study engineering. V.B. contributed to algorithm development and engineering. A.H.C. contributed to the writing of the manuscript and the study's medical perspective. All authors reviewed the manuscript and approved the final revision.
References

Digit Biomark 2021;5:37-43 DOI: $10.1159 / 000513222$
1 National Aeronautics and Space Administration. Apollo 11 Mission Report. Available from: https://www.nasa.gov/specials/apollo50th/pdf/A11_MissionReport.pdf.

2 Jacobs PL, Olvey SE. Metabolic and heart rate responses to open-wheel automobile road racing: a single-subject study. J Strength Cond Res. 2000;14(2):157-61.

3 Schwaberger G. Heart rate, metabolic and hormonal responses to maximal psychoemotional and physical stress in motor car racing drivers. Int Arch Occup Environ Health. 1987;59(6):579-604. 
4 Yanagida R, Takahashi K, Miura M, Nomura M, Ogawa Y, Aoki K, et al. Speed ratio but cabin temperature positively correlated with increased heart rates among professional drivers during car races. Environ Health Prev Med. 2016 Nov;21(6):439-45.

5 Carter JR, Kupiers NT, Ray CA. Neurovascular responses to mental stress. J Physiol. 2005 Apr;564(Pt 1):321-7.

6 Charkoudian N, Wallin BG. Sympathetic neural activity to the cardiovascular system: integrator of systemic physiology and interindividual characteristics. Compr Physiol. 2014 Apr;4(2):825-50.

7 Jones T, Williams B, Kilgallen C, Horobeanu C, Shillabeer B, Murray A, et al. A review of the performance requirements of squash. Int J Sports Sci Coaching. 2018;13(6):1223-32.

8 Kingsley M, James N, Kilduff LP, Dietzig RE, Dietzig B. An exercise protocol that simulates the activity patterns of elite junior squash. J Sports Sci. 2006 Dec;24(12):1291-6.
9 Li RT, Kling SR, Salata MJ, Cupp SA, Sheehan $\mathrm{J}$, Voos JE. Wearable performance devices in sports medicine. Sports Health. 2016 Jan-Feb; 8(1):74-8.

10 Sanders GJ, Boos B, Rhodes J, Kollock RO, Peacock CA. Competition-based heart rate, training load, and time played above $85 \%$ peak heart rate in NCAA Division I Women's Basketball. J Strength Cond Res. 2018, Online ahead of print.

11 Semin K, Stahlnecker Iv AC, Heelan K, Brown GA, Shaw BS, Shaw I. Discrepancy between training, competition and laboratory measures of maximum heart rate in NCAA division 2 distance runners. J Sports Sci Med. 2008 Dec;7(4):455-60.

12 Libs H, Boos B, Shipley F, Peacock CA, Sanders GJ. Variability in preseason jump loads and heart rate intensities in division I volleyball. Case Study. 2019;2(2):8.

13 Terbizan DJ, Dolezal BA, Albano C. Validity of seven commercially available heart rate monitors. Meas Phys Educ Exerc Sci. 2002; 6(4):243-7.

14 Pan J, Tompkins WJ. A real-time QRS detection algorithm. IEEE Trans Biomed Eng. 1985 Mar;32(3):230-6.
15 Boudet G, Garet M, Bedu M, Albuisson E, Chamoux A. Median maximal heart rate for heart rate calibration in different conditions: laboratory, field and competition. Int J Sports Med. 2002 May;23(4):290-7.

16 Coutinho C, Watson A, Brickson S, Sanfilippo J. Maximal heart rate differs between laboratory and field conditions among female athletes. J Hum Sport Exerc. 2017;12(2):386-95.

17 Nikolaidis. Maximal Heart Rate in Soccer Players: measured versus age-predicted. Biomed J. 2014;37(3).

18 Robergs RA, Landwehr R. The surprising history of the "HRmax=220-age" equation. J Exerc Physiol. 2002;5(2):1-10.

19 Schmitt L, Regnard J, Millet GP. Monitoring fatigue status with HRV measures in elite athletes: an avenue beyond RMSSD? Front Physiol. 2015 Nov;6:343.

20 Buchheit M. Monitoring training status with HR measures: do all roads lead to Rome? Front Physiol. 2014 Feb;5(73):73. 